\title{
Solving Frictional Contact Problems with Multigrid Efficiency
}

\author{
Konstantin Fackeldey ${ }^{1}$ and Rolf H. Krause ${ }^{1}$ \\ Institut für Numerische Simulation \\ Wegelerstr. 6, 53115 Bonn. e-mail: \{fackeldey, krause\}@ins.uni-bonn.de
}

\section{Introduction}

The construction of fast and reliable solvers for contact problems with friction is even nowadays a challenging task. It is well known that contact problems with Coulomb friction have the weak form of a quasi-variational inequality [KO88, HHNL88, NJH80]. For small coefficients of friction, a solution can be obtained by means of a fixed point iteration in the boundary stresses [NJH80]. This fixed point approach is often used for the construction of numerical methods, since in each iteration step only a constrained convex minimization problem has to be solved [DHK02, LPR91]. Unfortunately, the convergence speed of the discrete fixed point iteration deteriorates for smaller meshsizes. Here, we present a nonlinear multigrid method which removes the outer fixed point iteration and gives rise to a highly efficient solution method for frictional contact problems with Coulomb friction and other local friction laws in 2 and 3 space dimensions. The numerical cost is comparable to frictionless contact problems. Our method is based on monotone multigrid methods, see [KK01], and does not require any regularization of the non penetration condition or of the friction law. Therefore, the results are highly accurate. Using the basis transformation given in [WK00], our method can also be applied to two body contact problems.

\section{Elastic Contact with Coulomb Friction}

In this section, we give the strong and the weak formulation of the contact problem with Coulomb friction between a deformable body and a rigid foundation. We identify the body in its reference configuration with the domain $\Omega \subset \mathbb{R}^{d}, d=2,3$. The boundary $\partial \Omega$ is decomposed into three disjoint parts, $\Gamma_{D}, \Gamma_{N}$, and $\Gamma_{C}$, the possible contact boundary. The actual zone of contact is assumed to be contained in $\Gamma_{C}$ but is not known in advance. We assume 
$\operatorname{meas}_{d-1}\left(\Gamma_{D}\right)>0$ and denote tensor and vector quantities by bold symbols, e.g., $\boldsymbol{v}$, and the components by $v_{i}, 1 \leq i, j \leq d$ and $(\cdot)_{, j}=\partial / \partial x_{j}(\cdot)$. The summation convention is enforced on indices $1 \leq i, j \leq d$. We define the usual Sobolev space of displacements with weak derivative in $\boldsymbol{L}^{2}$ by $\boldsymbol{H}^{1}(\Omega):=\left(H^{1}(\Omega)\right)^{d}$ and set $\boldsymbol{H}_{D}:=\left\{v \mid v \in \boldsymbol{H}^{1}(\Omega), v_{\left.\right|_{\Gamma_{D}}}=\mathbf{0}\right\}$. We consider linear elastic material, i.e., the stresses $\boldsymbol{\sigma}=\left(\sigma_{i j}\right)_{i, j=1}^{d}$ are given by Hooke's law $\sigma_{i j}(\boldsymbol{u}):=E_{i j m l} u_{l, m}$. Here, Hooke's tensor $\mathbf{E}=\left(E_{i j m l}\right)_{i, j, l, m=1}^{d}$, $E_{i j l m} \in L^{\infty}(\Omega), 1 \leq i, j, l, m \leq d$ is sufficiently smooth, symmetric and positive definite. On $\partial \Omega$ the normal and tangential displacements are defined by $u_{n}=\boldsymbol{u} \cdot \boldsymbol{n}$ and $\boldsymbol{u}_{T}=\boldsymbol{u}-u_{n} \cdot \boldsymbol{n}$, where $\boldsymbol{n}$ is the outer normal vector. Similarly, $\sigma_{n}=n_{i} \sigma_{i j} n_{j}$ and $\left(\boldsymbol{\sigma}_{T}\right)_{i}=\sigma_{i j} n_{j}-\sigma_{n} \cdot \boldsymbol{n}$ are the normal and tangential stresses, respectively. Let $g: \mathbb{R}^{d} \supset \Gamma_{C} \rightarrow \mathbb{R}$ be a a continuous function giving the distance between the body and the rigid foundation, taken in normal direction with respect to the reference configuration. Then, for small deformations, we can say that the body $\Omega$ does not penetrate the rigid foundation if we have $u_{n}(x) \leq g(x)$ for all $x \in \Gamma_{C}$, see e.g., [KO88].

At the points, where the body and the rigid foundation come into contact, friction may occur. Here we use the Coulomb law of friction. It states that the force, which is needed to move a body lengthwise a rigid foundation, is proportional to the force pushing the body perpendicular onto the rigid foundation. The boundary value problem constituting the elastic contact problem with friction consists of the equilibrium condition (1) in $\Omega$, the boundary conditions (2) and (3) on $\Gamma_{D}$ and $\Gamma_{N}$, the contact conditions (5), (6) on $\Gamma_{C}$ and the Coulomb law of friction $(7),(8)$ on $\Gamma_{C}$. In equations $(7),(8),|\cdot|$ is the Euclidean norm on $\mathbb{R}^{d-1}$. Here, we assume sufficiently smooth data and the for coefficient of friction holds $\mathcal{F} \in L^{\infty}\left(\Gamma_{C}\right)$ and $\mathcal{F} \geq \mathcal{F}_{0}>0$ a.e. on $\Gamma_{C}$.

$$
\begin{array}{rlrlrl}
-\sigma_{i j}(\boldsymbol{u})_{, j} & =f_{i} & \text { in } \Omega & (1) & \boldsymbol{u} \cdot \boldsymbol{n} & \leq g \\
\boldsymbol{u} & =0 & \text { on } \Gamma_{D} & (2) & (\boldsymbol{u} \cdot \boldsymbol{n}-g) \sigma_{n}=0 \\
\sigma_{i j}(\boldsymbol{u}) \cdot n_{j} & =p_{i} & \text { on } \Gamma_{N} & (3) & \boldsymbol{u}_{T}=0 \Rightarrow\left|\boldsymbol{\sigma}_{T}\right|<\mathcal{F}\left|\sigma_{n}\right| \\
\sigma_{n} & \leq 0 & & (4) & \boldsymbol{u}_{T} \neq 0 \Rightarrow \boldsymbol{\sigma}_{T}=-\mathcal{F}\left|\sigma_{n}\right| \frac{\boldsymbol{u}_{T}}{\left|\boldsymbol{u}_{T}\right|}
\end{array}
$$

By (7) and (8), Coulomb's law of friction is a local friction law, since the frictional response at $x \in \Gamma_{C}$ depends only on the stress developed at $x$. Moreover, we can divide all points in the actual zone of contact into sticking and sliding points. A point $x$ is called sticky, if no tangential displacement occurs, i.e., if $\boldsymbol{u}_{T}(x)=\mathbf{0}$. It is called sliding, if $\boldsymbol{u}_{T}(x) \neq \mathbf{0}$. Of particular interest is boundary condition (8). For $d=2$ and $\boldsymbol{u}_{T} \neq 0$, we have $\boldsymbol{u}_{T} /\left|\boldsymbol{u}_{T}\right| \in\{-1,1\}$. This is in contrast to the case $d=3$, where $\boldsymbol{u}_{T} /\left|\boldsymbol{u}_{T}\right| \in S^{2}=\left\{\boldsymbol{v}\left|\boldsymbol{v} \in \mathbb{R}^{2},\right| \boldsymbol{v} \mid=1\right\}$. In order to give the variational formulation of problem (1)-(8), let us define the closed convex set $\mathcal{K}$ of admissible displacements

$$
\mathcal{K}:=\left\{\boldsymbol{v} \in \boldsymbol{H}_{D} \mid v_{n} \leq g \text { on } \Gamma_{C}\right\} .
$$


We define the bilinear form $a(\cdot, \cdot)$ by $a(\boldsymbol{u}, \boldsymbol{v})=\int_{\Omega} \sigma_{i j}(\boldsymbol{u}) v_{i, j} d x$ and set $f(\boldsymbol{v})=\int_{\Omega} f_{i} v_{i} d x+\int_{\Gamma_{N}} p_{i} v_{i} d s$. At the contact boundary the virtual work of the frictional forces is characterized by the following nonlinear and nondifferentiable functional $j: \boldsymbol{H}_{D} \times \boldsymbol{H}_{D} \rightarrow \mathbb{R}$

$$
j(\boldsymbol{u}, \boldsymbol{v})=\int_{\Gamma_{C}} \mathcal{F}\left|\sigma_{n}(\boldsymbol{u})\right|\left|\boldsymbol{v}_{T}\right| d s .
$$

Using these definitions, the weak formulation of the boundary value problem (1)-(8) is given by the following quasi-variational inequality: Find $\boldsymbol{u} \in \mathcal{K}$, such that

$$
a(\boldsymbol{u}, \boldsymbol{v}-\boldsymbol{u})+j(\boldsymbol{u}, \boldsymbol{v})-j(\boldsymbol{u}, \boldsymbol{u}) \geq f(\boldsymbol{v}-\boldsymbol{u}), \quad \boldsymbol{v} \in \mathcal{K},
$$

see [DL76, KO88, HHNL88]. The functional $j$ is nonconvex, nonquadratic and nondifferentiable. Thus, standard methods from convex analysis cannot be applied to gain a solution of the quasi-variational inequality (11).

\section{A Multigrid Method for a quasi-variational Inequality}

In [NJH80, HHNL88], the following fixed point iteration is considered: Let $\boldsymbol{u}^{0} \in \mathcal{K}$ be given. Then, for $k=1,2, \ldots$, compute $\boldsymbol{u}^{k}$ as the unique solution of the variational inequality

$$
a\left(\boldsymbol{u}^{k}, \boldsymbol{v}-\boldsymbol{u}^{k}\right)+j\left(\boldsymbol{u}^{k-1}, \boldsymbol{v}\right)-j\left(\boldsymbol{u}^{k-1}, \boldsymbol{u}^{k}\right) \geq f\left(\boldsymbol{v}-\boldsymbol{u}^{k}\right) \quad \boldsymbol{v} \in \mathcal{K} .
$$

Setting $\tau=-\sigma_{n}\left(\boldsymbol{u}^{k-1}\right)$ and introducing

$$
H_{+}^{-1 / 2}:=\left\{v \in H^{-1 / 2}\left(\Gamma_{C}\right) \mid\langle v, w\rangle_{H^{-1 / 2} \times H^{1 / 2}} \geq 0, \quad w \in H^{1 / 2}\left(\Gamma_{C}\right)\right\},
$$

(12) defines a mapping $\Psi: H_{+}^{-1 / 2} \rightarrow H_{+}^{-1 / 2}$ by $\Psi(\tau)=-\sigma_{n}\left(\boldsymbol{u}^{k}\right)$. For sufficiently small $\mathcal{F}$, this mapping can be shown to have a fixed point, which is a solution of problem (11), see [NJH80, HHNL88]. This fixed point iteration can be used for the numerical solution of (11), see [LPR91, Kra01, DHK02, Hil02] and is also the starting point for our method.

In this section, we present a nonlinear multigrid method by means of which the variational inequality (12) can be solved efficiently. Moreover, we extend our method in a way that the fixed point iteration is removed. The resulting nonlinear multigrid method can then be applied to the quasi-variational inequality (11) directly. In our numerical experiments, see Section 4, this method has been shown to be an iterative solution method for (11) with multigrid complexity.

Let $\left(\mathcal{T}_{\ell}\right)_{\ell=1}^{L}$ denote a family of nested and shape regular meshes with meshsize parameter $h_{\ell}$. We use Lagrangian conforming finite elements $\boldsymbol{S}_{\ell} \subset \boldsymbol{H}_{D}$ of first order. The set of nodes of $\mathcal{T}_{\ell}$ is denoted by $\mathcal{N}^{(\ell)}$ and the nodal basis functions of $S_{\ell}$ are $\left\{\lambda_{p}^{(\ell)}\right\}_{p \in \mathcal{N}^{(\ell)}}$. The nodes on the possible contact boundary are $\mathcal{C}^{(\ell)}=\Gamma_{C} \cap \mathcal{N}^{(\ell)}$. As discretization of the convex set $\mathcal{K}$ we take 


$$
\mathcal{K}_{L}:=\left\{\boldsymbol{u} \in \boldsymbol{S}_{L} \mid \boldsymbol{u}(p) \cdot \boldsymbol{n}(p) \leq g(p), p \in \mathcal{C}^{(L)}\right\} .
$$

Note, that in general $\mathcal{K}_{L} \not \subset \mathcal{K}$. We define the discrete normal stresses $s_{n}\left(\boldsymbol{u}_{L}\right)$ for $\boldsymbol{u} \in \boldsymbol{S}_{L}$ on the basis of Greens theorem as the linear residual by

$$
\left(s_{n}(\boldsymbol{u})\right)_{p}:=r\left(\lambda_{p}^{(L)} \cdot \boldsymbol{n}\right):=a\left(\boldsymbol{u}, \lambda_{p}^{(L)} \cdot \boldsymbol{n}\right)-\boldsymbol{f}\left(\lambda_{p}^{(L)} \cdot \boldsymbol{n}\right),
$$

cf. [HHNL88]. As discretization for the functional $j$ we choose for $\boldsymbol{u}, \boldsymbol{v} \in \boldsymbol{S}_{L}$

$$
j^{L}(\boldsymbol{u}, \boldsymbol{v}):=\sum_{p \in \mathcal{C}^{(L)}} \mathcal{F}\left|\left(s_{n}(\boldsymbol{u})\right)_{p}\right|\left|\boldsymbol{v}_{T}(p)\right| d s
$$

Inserting the functional $j^{L},(12)$ gives rise to the discrete fixed point iteration: Let $\boldsymbol{u}^{0} \in \boldsymbol{S}_{L}$ be given. For $k=1,2, \ldots$ solve

$$
a\left(\boldsymbol{u}^{k}, \boldsymbol{v}-\boldsymbol{u}^{k}\right)+j^{L}\left(\boldsymbol{u}^{k-1}, \boldsymbol{v}\right)-j^{L}\left(\boldsymbol{u}^{k-1}, \boldsymbol{u}^{k}\right) \geq f\left(\boldsymbol{v}-\boldsymbol{u}^{k}\right), \quad \boldsymbol{v} \in \mathcal{K}_{L},
$$

in each step of which a variational inequality has to be solved. We remark that for the contractivity constant $C$ of the discrete fixed point iteration (15) holds $C=C(h)=\mathcal{O}\left(h^{-1 / 2}\right)$, see [Hil02]. Thus, the iteration process slows down for decreasing meshsize $h$.

As starting point for our nonlinear multigrid method let us note that the solution $\boldsymbol{u}^{k}$ of (12) can equivalently be characterized as the unique minimizer of the convex functional

$$
\hat{\mathcal{J}}_{\boldsymbol{u}^{k-1}}(\cdot)=J+j\left(\boldsymbol{u}^{k-1}, \cdot\right)+\varphi_{\mathcal{K}}=\left(\frac{1}{2} a(\cdot, \cdot)-f(\cdot)\right)+j\left(\boldsymbol{u}^{k-1}, \cdot\right)+\varphi_{\mathcal{K}}
$$

over $\boldsymbol{H}_{D}$. Here, $\varphi_{\mathcal{K}}$ is defined by $\varphi_{\mathcal{K}}(\boldsymbol{u})=+\infty$ for $\boldsymbol{u} \notin \mathcal{K}$ and zero for $\boldsymbol{u} \in \mathcal{K}$. As discretization of $\hat{\mathcal{J}}_{\boldsymbol{u}^{k-1}}$ we choose on the basis of (14) the functional $\hat{\mathcal{J}}_{\boldsymbol{u}^{k-1}}^{L}:=J+j^{L}\left(\boldsymbol{u}^{k-1}, \cdot\right)+\varphi_{\mathcal{K}_{L}}$. Following [Kor97, KK01], we seek the minimizer of $\hat{\mathcal{J}}_{\boldsymbol{u}^{k-1}}^{L}$ over $\boldsymbol{S}_{L}$ by successive minimization in direction of a suitable multilevel basis. To this end, we associate with each node $p \in \mathcal{N}^{(\ell)}$, $1 \leq \ell \leq L$, the local subspace $\boldsymbol{V}_{p}=\operatorname{span}\left\{\lambda_{p}^{(\ell)} \cdot \boldsymbol{e}_{1}(p), \ldots, \lambda_{p}^{(\ell)} \cdot \boldsymbol{e}_{d}(p)\right\}$. For the nodes $p \in \mathcal{C}^{(L)}$ we choose $\boldsymbol{e}_{1}(p)=\boldsymbol{n}(p)$ and extend $\boldsymbol{e}_{1}(p)$ to an orthonormal basis of $\mathbb{R}^{d}$. For all remaining nodes we choose $\left\{\boldsymbol{e}_{i}(p)\right\}_{1 \leq i \leq d}$ to be the canonical basis vectors of $\mathbb{R}^{d}$ and set $\boldsymbol{V}_{p}=\operatorname{span}\left\{\mu_{p}^{(\ell)} \cdot \boldsymbol{e}_{1}(p), \ldots, \mu_{p}^{(\ell)} \cdot \boldsymbol{e}_{d}(p)\right\}$ with functions $\mu_{p}^{(\ell)}$ to be discussed later. We moreover assume an ordering $k=k(p, \ell)$ of all nodes on all levels to be given such that $k(p, r) \leq k(q, s)$ implies $r \geq s$ for $p, q \in \mathcal{N}^{(\ell)}, 1 \leq \ell \leq L$. Now, we can introduce the multilevel splitting

$$
\mathcal{S}^{(L)}=\boldsymbol{V}_{p_{1}}+\cdots+\boldsymbol{V}_{p_{n_{L}}}+\boldsymbol{V}_{p_{n_{L}+1}}+\cdots+\boldsymbol{V}_{p_{M}}
$$

where we have set $n_{L}=\# \mathcal{N}^{(L)}$ and the indices $n_{L}+1, \ldots, M$ stand for the coarse grid corrections.

We first consider the successive minimization of $\hat{\mathcal{J}}_{\boldsymbol{u}^{k-1}}^{L}$ with respect to the leading subspaces $\boldsymbol{V}_{p_{1}}+\cdots+\boldsymbol{V}_{p_{n_{L}}}$. Let $\boldsymbol{w}^{0} \in \mathcal{S}_{L}$ be given and set $\boldsymbol{w}_{0}=\boldsymbol{w}^{0}$. 
Then, for $1 \leq m \leq n_{L}$, the local minimization problem: find $\boldsymbol{v}_{m} \in \boldsymbol{V}_{p_{m}}$, such that

$$
\hat{\mathcal{J}}_{\boldsymbol{u}^{k-1}}^{L}\left(\boldsymbol{v}_{m}+\boldsymbol{w}_{m-1}\right) \leq \hat{\mathcal{J}}_{\boldsymbol{u}^{k-1}}^{L}\left(\boldsymbol{v}+\boldsymbol{w}_{m-1}\right), \quad \boldsymbol{v} \in \boldsymbol{V}_{p_{m}},
$$

is solved and update $\boldsymbol{w}_{m}=\boldsymbol{w}_{m-1}+\boldsymbol{v}_{m}$. Setting $\boldsymbol{w}^{1}:=\boldsymbol{w}_{n_{L}}$, by this nonlinear Gauß-Seidel method a sequence $\boldsymbol{w}^{\nu}, \nu=0,1, \ldots$, of iterates is defined which converges to the unique minimizer of $\hat{\mathcal{J}}_{\boldsymbol{u}^{k-1}}^{L}$, see [Glo84, KK01] and [JAJ98] for a related method using regularization. We refer to [Kra] for a detailed description how the local problems (17) can be solved efficiently.

The convergent but slow iteration (17) is now accelerated by additional minimization steps in the direction of coarse grid functions $\boldsymbol{\mu}_{p}^{(l)}, l<L$, with larger support. In contrast to multigrid methods for linear problems, we cannot represent the functional $\hat{\mathcal{J}}_{\boldsymbol{u}^{k-1}}^{L}$ to be minimized on the coarser grids and therefore have to use nonlinear coarse grid corrections.

To this end, we introduce the smoothed fine grid iterate $\overline{\boldsymbol{u}}_{L}:=\boldsymbol{w}^{\nu}$, which is obtained after $\nu>0$ presmoothing steps of the Gauß-Seidel method (17). Since the functional $\varphi_{\mathcal{K}_{L}}+j^{L}\left(\boldsymbol{u}^{k-1}, \cdot\right)$ is nondifferentiable and nonquadratic, we restrict the coarse grid corrections to a neighborhood of the fine grid iterate $\overline{\boldsymbol{u}}_{L}$ where the functional $\hat{\mathcal{J}}_{\boldsymbol{u}^{k-1}}^{L}$ is smooth and can be linearized, see [Kor02]. We define the neighbourhood $\mathcal{K}_{\overline{\boldsymbol{u}}_{L}}$ of the iterate $\overline{\boldsymbol{u}}_{L}$ by

$\mathcal{K}_{\overline{\boldsymbol{u}}_{L}}=\left\{\boldsymbol{w} \in \mathcal{K}_{L} \mid w_{n}(p)=0, p\right.$ sticky; $(\boldsymbol{w})_{T} \lessgtr-\left(\overline{\boldsymbol{u}}_{L}\right)_{T}$, if $\left(\overline{\boldsymbol{u}}_{L}\right)_{T} \lessgtr 0$ and $p$ sliding $\}$.

The inequalities have to be understood component wise. On $\mathcal{K}_{\overline{\boldsymbol{u}}_{L}}$, we define

$$
j_{\overline{\boldsymbol{u}}_{L}}(\boldsymbol{w})=\sum_{\left\{p \in \mathcal{C}^{(L)} \mid \overline{\boldsymbol{u}}_{L}(p) \cdot \boldsymbol{n}(p)=g(p)\right\}} \mathcal{F}\left|\left(s_{n}\left(\boldsymbol{u}^{k-1}\right)\right)_{p}\right|\left|\boldsymbol{w}_{T}(p)\right|,
$$

which is a smooth functional, and the quadratic energy functional $\overline{\mathcal{J}}_{\overline{\boldsymbol{u}}_{L}}^{L}$ by

$$
\overline{\mathcal{J}}_{\overline{\boldsymbol{u}}_{L}}^{L}=\frac{1}{2}\left(a(\cdot, \cdot)+j_{\overline{\boldsymbol{u}}_{L}}^{\prime \prime}\left(\overline{\boldsymbol{u}}_{L}\right)(\cdot, \cdot)\right)-\left(f(\cdot)-j_{\overline{\boldsymbol{u}}_{L}}^{\prime}\left(\overline{\boldsymbol{u}}_{L}\right)(\cdot)+j_{\overline{\boldsymbol{u}}_{L}}^{\prime \prime}\left(\overline{\boldsymbol{u}}_{L}\right)\left(\overline{\boldsymbol{u}}_{L}, \cdot\right)\right) .
$$

The resulting quadratic obstacle problem: Find $\boldsymbol{c} \in \mathcal{K}_{\overline{\boldsymbol{u}}_{L}}$, such that

$$
\overline{\mathcal{J}}_{\overline{\boldsymbol{u}}_{L}}^{L}(\boldsymbol{c}) \leq \overline{\mathcal{J}}_{\overline{\boldsymbol{u}}_{L}}^{L}(\boldsymbol{v}), \quad \boldsymbol{v} \in \mathcal{K}_{\overline{\boldsymbol{u}}_{L}}
$$

requires an obstacle problem with constraints in both, normal and tangential direction, to be solved. These constraints stem from the non penetration condition and the friction law, respectively. The additional minimization steps for $m>n_{L}$ of our splitting (16) are now done with respect to the energy (18). In order to improve the convergence speed of our method, we use so called truncated basis functions $\boldsymbol{\mu}_{p}^{(\ell)}, \ell<L$, which allow for representing the actual guessed contact boundary on coarser grids, see [Kor97, KK01]. The resulting multigrid method can be implemented as a $\mathcal{V}$-cycle and is globally convergent, see [KK01, Kra01]. It does not require any regularization, neither of the non penetration condition nor of the functional $j$. Moreover, no algorithmic parameters as damping or regularization parameters have to be chosen. We now present the two algorithms to be compared in the next section. 
Algorithm 1 (Fixed point iteration with multigrid method) Initialize: $\boldsymbol{u}^{0}=\mathbf{0} . \quad$ for $k=1, \ldots, k \max$ do

Find $\boldsymbol{u}^{k} \in \mathcal{K}_{L}$ by applying sufficiently many multigrid steps to: $a\left(\boldsymbol{u}^{k}, \boldsymbol{v}-\boldsymbol{u}^{k}\right)+j^{L}\left(\boldsymbol{u}^{k-1}, \boldsymbol{v}\right)-j^{L}\left(\boldsymbol{u}^{k-1}, \boldsymbol{u}^{k}\right) \geq f\left(\boldsymbol{v}-\boldsymbol{u}^{k}\right), \quad \boldsymbol{v} \in \mathcal{K}_{L}$.

if $\left|s_{n}\left(\boldsymbol{u}^{k-1}\right)-s_{n}\left(\boldsymbol{u}^{k}\right)\right| /\left|s_{n}\left(\boldsymbol{u}^{k}\right)\right| \leq$ TOL break

Compute the normal stress $s_{n}\left(\boldsymbol{u}^{k}\right)$ as linear residual. end

Algorithm 2 (Nonlinear multigrid method)

Initialize: $\boldsymbol{w}^{0}=\boldsymbol{u}^{0}=\mathbf{0}$.

Find $\boldsymbol{u}_{L} \in \mathcal{K}_{L}$ by doing sufficiently many of the following multigrid steps:

for $m=1, \ldots, n_{L}$ do

Find $\boldsymbol{v}_{m} \in \boldsymbol{V}_{p_{m}}$, such that

$\hat{\mathcal{J}}_{\boldsymbol{w}_{m-1}}^{L}\left(\boldsymbol{v}_{m}+\boldsymbol{w}_{m-1}\right) \leq \hat{\mathcal{J}}_{\boldsymbol{w}_{m-1}}^{L}\left(\boldsymbol{v}+\boldsymbol{w}_{m-1}\right), \quad \boldsymbol{v} \in \boldsymbol{V}_{p_{m}}$.

Update $\boldsymbol{w}_{m}=\boldsymbol{w}_{m-1}+\boldsymbol{v}_{m}$.

end

Set $\overline{\boldsymbol{u}}_{L}=\boldsymbol{w}_{n_{L}}$ and compute coarse grid correction $\boldsymbol{c}$ with respect to $\overline{\mathcal{J}}_{\overline{\boldsymbol{u}}_{L}}$

Set $\boldsymbol{u}_{L}=\overline{\boldsymbol{u}}_{L}+c$

Algorithm 2 constitutes our nonlinear multigrid method for the quasi-variational inequality (11). It has shown to converge in all of our numerical experiments, even then, when the exact fixed point iteration method fails, see Section 4 .

\section{Numerical Results}

In this section we present numerical results for a Hertzian contact problem in two space dimensions. We consider a half circle in contact with a rigid foundation. The half circle is centered at $(0,0.4)$ with radius 0.4 and we have chosen $E=2000$ and $\nu=0.28$ as material parameters. We prescribe vertical displacement $u(x, y)=-0.005$ at the upper boundary $\Gamma_{D}=\{(x, y) \in \Gamma \mid y=0.4\}$ and set $\Gamma_{C}=\partial \Omega \backslash \Gamma_{D}$ and $\boldsymbol{n}(p)=(0,-1)^{T}$ for $p \in \mathcal{C}^{(\ell)}$. We discretize with linear and bilinear finite elements on triangles and quadrilaterals, respectively and chose $\mathcal{T}_{1}$ as depicted in the left of Figure 2. A multilevel hierarchy is created up to Level $L=11$ by successive adaptive refinement. We now investigate the convergence properties of Algorithm 1 and of our Algorithm 2. In Table 1 and the right picture in Figure 1, the number of outer iteration steps of Algorithm 1 is given. Here, we have set $\mathrm{TOL}=10^{-9}$ in Algorithm 1. As expected from the theory, for large coefficients of friction and small meshsize parameters we have no convergence of Algorithm 1. Note, that for $\mathcal{F}=5.0$ the nonlinear multigrid method 2 converges even for small meshsizes. In order to compare the total amount of work needed using Algorithm 1 and Algorithm 2, respectively, we compare the total number of $\mathcal{V}$ - cycles needed to obtain a solution of (12) on each level. For the fixed point iteration we sum up the number of $\mathcal{V}$-Cycles in each iteration step per Level. For our multigrid method we simply take the total number of iterations. In both cases the multigrid iteration is stopped, if 


\begin{tabular}{|l|l|l|l|l|l|l||l|l|l|l|l|}
\hline $\mathcal{F} \backslash \#$ dof & 94 & 830 & 4.674 & 44.200 & 334.818 & $\mathcal{F} \backslash \#$ dof & 94 & 830 & 4.674 & 4.4200 & 334.818 \\
\hline \hline 0.08 & 1 & 5 & 6 & 6 & 6 & 1.1 & 1 & 6 & 10 & 16 & 18 \\
\hline 0.4 & 1 & 5 & 10 & 10 & 10 & 2.0 & 1 & 6 & 8 & 19 & 33 \\
\hline 0.7 & 1 & 5 & 8 & 12 & 13 & 5.0 & 1 & 2 & 2 & no conv. & no conv. \\
\hline 0.9 & 1 & 6 & 9 & 15 & 15 & 8.0 & 1 & 2 & 2 & no conv. & no conv. \\
\hline 1.0 & 1 & 6 & 10 & 10 & 16 & 40.0 & 1 & 2 & 2 & no conv. & no conv. \\
\hline
\end{tabular}

Table 1. Number of exact fixed point iterations on Level $\ell=3,5,7,9,11$

for two two consecutive iterates $\boldsymbol{u}^{\nu}, \boldsymbol{u}^{\nu+1}$ holds $\left\|\boldsymbol{u}^{\nu}-\tilde{\boldsymbol{u}}^{\nu+1}\right\|_{a} \leq 10^{-11}$ with $\|\boldsymbol{u}\|_{a}^{2}=a(\boldsymbol{u}, \boldsymbol{u})$. As starting value we always use $\boldsymbol{u}^{0}=0$. As can be seen from
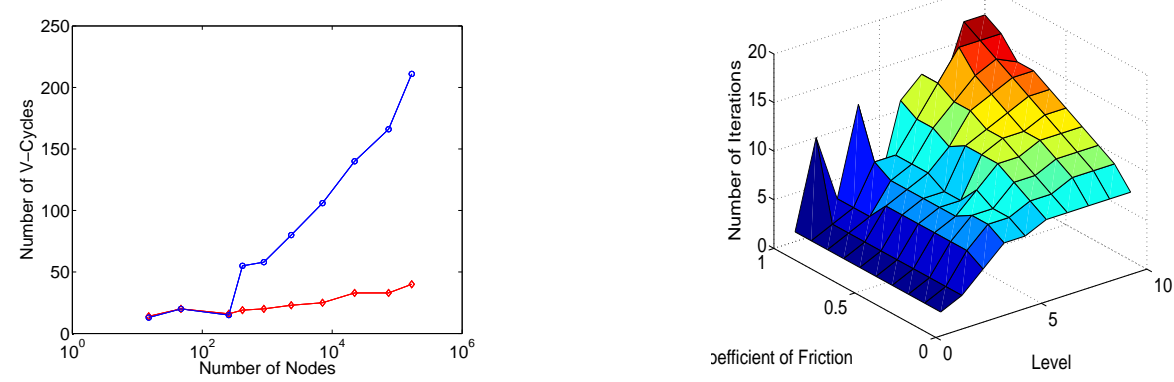

Fig. 1. Left: Comparison of exact fixed point iteration Algorithm 1 and nonlinear multigrid method Algorithm 2. Right: Behavior of the fixed point iteration

the left picture in Figure 1, our nonlinear methods shows multigrid efficiency (red line) and is of much higher efficiency than the fixed point iteration (blue line). The number of needed $V$-cycles is independet of $h$.

Finally, the middle picture of Figure 2 shows the tangential (red) and normal stresses (blue) for $\mathcal{F}=0.4$. As can be seen, sliding and sticky nodes are clearly identificated by our method. The implementation of our method has
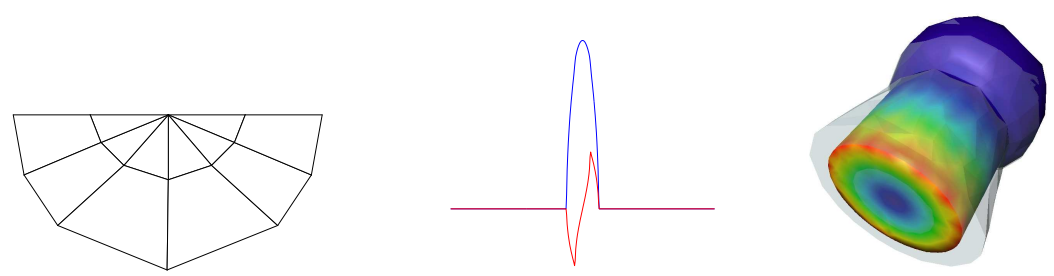

Fig. 2. Left: $\mathcal{T}_{1}$ Middle: Boundary stress Right: $3 d$ example 
been done in the framework of the FEM-toolbox UG, see [BBJ $\left.{ }^{+} 97\right]$. Thus, it is also applicable to more complicated geometries and unstructured grids, as can be seen in the left Figure 2. As an example of a $3 d$ geometry, here the displacements of a deformed cork in frictional contact with a surrounding bottle are shown. The reference configuration is given by the shaded transparent surface. For a more detailed discussion of numerical results in $3 d$ and with elastic contact we refer to [Kra].

\section{References}

$\left[\mathrm{BBJ}^{+}\right.$97] P. Bastian, K. Birken, K. Johannsen, S. Lang, N. Neuß, H. RentzReichert, and C. Wieners. UG - a flexible software toolbox for solving partial differential equations. CVS, 1:27-40, 1997.

[DHK02] Z. Dostal, J. Haslinger, and R. Kučera. Implementation of the fixed point method in contact problems with Coulomb frcition based on a dual splitting type technique. J. Comp. and Appl. Math., 140:245-256, 2002.

[DL76] G. Duvaut and J.L. Lions. Inequalities in mechanics. Springer, 1976.

[Glo84] R. Glowinski. Numerical methods for nonlinear variational problems. Series in Computational Physics. Springer, New York, 1984.

[HHNL88] I. Hlaváček, J. Haslinger, J. Nečas, and J. Lovíšek. Solution of variational inequalities in mechanics. Springer, 1988.

[Hil02] P. Hild. On finite element uniqueness studies for Coulomb's frictional contact model. Int. J. Appl. Math. Comput. Sci., 12(1):41-50, 2002.

[JAJ98] F. Jourdan, P. Alart, and M. Jean. A Gauß-Seidel like algorithm to solve frictional contact problems. Comp. Meth. in Appl. Mech. Eng., pages 31-47, 1998.

[KK01] R. Kornhuber and R. H. Krause. Adaptive multigrid methods for Signorini's problem in linear elasticity. CVS, 4:9-20, 2001.

[KO88] N. Kikuchi and J.T. Oden. Contact problems in elasticity: a study of variational inequalities and finite element methods. SIAM, 1988.

[Kor97] R. Kornhuber. Adaptive Monotone Multigrid Methods for Nonlinear Variational Problems. Teubner, 1997.

[Kor02] R. Kornhuber. On constrained Newton linearization and multigrid for variational inequalities. Numerische Mathematik, 99:699-721, 2002.

[Kra] R. H. Krause. Efficient solution of frictional contact problems without regularization. in preparation.

[Kra01] R. H. Krause. Monotone Multigrid Methods for Signorini's Problem with Friction. PhD thesis, Freie Universität Berlin, 2001.

[LPR91] C. Licht, E. Pratt, and M. Raous. Remarks on a numerical method for unilateral contact including friction. Int. Ser. of Numerical Mathematics, 101:129-144, 1991.

[NJH80] J. Nečas, J. Jarušek, and J. Haslinger. On the solution of the variational inequality to the Signorini problem with small friction. Bollettino U.M.I., 17:796-811, 1980.

[WK00] B. Wohlmuth and R. H. Krause. Monotone methods on non-matching grids for non linear contact problems. SISC, Vol. 25:324-347, 2000. 